\title{
Applications of the Volume Averaging Theory to Momentum and Heat Transfer within Complex Flow Systems
}

\author{
Akira Nakayama *†
}

\section{Abstract}

The volume averaging theory (VAT) developed in the study of porous media is quite powerful in attacking difficult problems associate with momentum and heat transfer in complex fluid flow system, such as heat exchangers, combustors and engine nacelles. Applications of VAT to momentum and heat transfer within complex heat and flow systems are reviewed in this lecture. Such difficulties arise from geometrical complexities and conjugate heat transfer between fluids and solids. In order to overcome the difficulties, the set of the governing equations are integrated over a local control volume to obtain the macroscopic governing equations. The sub-scale (i.e. pore-scale) modeling is carried out to close the set of the equations. Subsequently, the unknown model constants are determined by conducting direct numerical simulations using a structural unit model. Various applications in heat exchangers, composting systems and human bodies are discussed to elucidate the validity of the present procedure.

* Department of Mechanical Engineering, Shizuoka University, Hamamatsu, Japan 432-8561

† School of Civil Engineering and Architecture, Wuhan Polytechnic University, Hubei, Wuhan, 430023 China 


\section{Introduction}

Difficulties arise when we conduct CFD calculations for complex structures as found in heat exchangers. A grid system, designed for a comparatively large scale of the shell (plates), would not be fine enough to describe the details of flow and heat transfer around tubes (fins). Furthermore, heat transfer from hot to cold fluids through conducting solid structures makes the problem highly conjugate and thus formidable. Under these situations, the concept of local volume-averaging theory, namely, VAT, widely used in the study of porous media [Cheng [1], Quintard and Whitaker [2], Nakayama [3]] may be quite useful. We shall seek such a general numerical procedure to investigate the flow and heat transfer within complex heat and fluid flow equipment.



Figure 1. Schematic view of heat exchanger

In VAT, we use a grid system, which is just fine enough to resolve macroscopic flow and temperature fields. We do not grid smallscale elements but model them as a porous medium. For example, hot and cold fluid passages in a heat exchanger, as illustrated in Figure 1, may be treated as two distinct porous media of highly anisotropic permeabilities, separated by solid walls. Perhaps, we have no other choice but to appeal to a volume-averaged set of 
equations with some subscale model, when performing CFD calculations of engine nacelles to account for bundles of hydraulic tubes, ribs and some other obstructions [DesJardin [4]]. There are a number of other situations in which we need to introduce a volume averaged set of macroscopic equations and its subscale models to describe complex equipment.

In order to apply these macroscopic governing equations based on VAT such as introduced by Nakayama et al. [5] for analyzing the complex heat and fluid flow equipment, we must model the flow resistance associated with subscale solid elements (modeled as an anisotropic porous medium) and also the heat transfer between the flowing fluid and the subscale elements, beforehand. Such subscale modeling can be made performing microscopic (pore scale) numerical calculations on individual small elements (such as fins and bundles of hydraulic tubes). The microscopic numerical results thus obtained at a pore scale can be processed to extract the macroscopic hydrodynamic and thermal characteristics in terms of the volume-averaged quantities. A great deal of effort has been directed towards this endeavor [Nakayama and Kuwahara [6]].

In the fundamental study of turbulent flow through an isotropic porous medium, Nakayama and Kuwahara [7] pointed out that the smaller eddies (i.e. turbulent mixing rather than mechanical dispersion) must be modeled first, as in the case of LES, and started with the Reynolds averaged set of the governing equations and integrated them over a representative control volume to obtain the set of macroscopic equations, namely, the equations of continuity, momentum, energy, turbulence kinetic energy and its rate of dissipation. The unknown model parameters including the interfacial heat transfer coefficient, permeability and Forchheimer constants were determined by carrying out exhaustive numerical experiments using a periodic array of square rods and then integrating these microscopic results over a unit porous structure [Nakayama and Kuwahara [7]]. Their study can be generalized to treat anisotropic porous media, which represent complex heat and fluid flow equipment consisting of synthetic structural elements. Possible steps based on the theory of porous media for analyzing complex heat transfer equipment may be summarized as follows: 
- $1^{\text {st }}$ step: Derive a set of volume averaged governing equations by integrating the set of Reynolds averaged conservation equations for mass, momentum, energy and turbulence quantities over a local control-volume.

- $2^{\text {nd }}$ step: Establish possible subscale models for unknown terms associated with deviations from averaged values, namely, permeability tensor, Forcheimer tensor, and interfacial heat transfer coefficient.

- 3 rd step: Determine all model constants by conducting microscopic numerical simulations over individual elements.

- $4^{\text {th }}$ step: Solve the set of volume averaged governing equations with the subscale model equations and their model constants just determined.

\section{Volume Averaged Governing Equations}

In most applications associated with heat exchangers, the fluids do not mix but transfer heat through a separating wall, as illustrated in Figure 1. Therefore, we must consider the two sets of the microscopic governing equations for both hot and cold fluids. Each set of the microscopic governing equations consists of the Reynolds averaged continuity equation, Navier-Stokes equation, energy equations, the transport equation of turbulence kinetic energy and that of its dissipation rate. We decompose the Reynolds averaged variable into its volume averaged component and the deviation from it, and integrate the two sets of Reynolds averaged equations for the hot and cold fluid phases and the heat conduction equation for the solid phase over a control volume $V$, which is much larger than a microscopic (clutter) characteristic size but much smaller than a macroscopic characteristic size. The resulting macroscopic equations naturally yield a number of correlations among the spatially deviating components. Nakayama and Kuwahara [7] mathematically modeled these unknown terms (correlations) to close the set of macroscopic equations for the case of isotropic 4 
Applications of the Volume Averaging Theory $\quad$ Mapana J Sci, 11, 3(2012)

porous media. Their mathematical treatment can be generalized for the case of anisotropic porous media as follows:

$$
\begin{gathered}
\frac{\partial}{\partial x_{j}}\left\langle\overline{u_{j}}\right\rangle^{f}=0 \\
\frac{\partial\left\langle\overline{u_{i}}\right\rangle^{f}}{\partial t}+\frac{\partial}{\partial x_{j}}\left\langle\overline{u_{j}}\right\rangle^{f}\left\langle\bar{u}_{i}\right\rangle^{f}=-\frac{1}{\rho_{f}} \frac{\partial}{\partial x_{i}}\left(\langle\bar{p}\rangle^{f}+\frac{2}{3} \rho_{f}\langle k\rangle^{f}\right) \\
+\frac{\partial}{\partial x_{j}}\left[\left(v+v_{t}\right)_{f}\left(\frac{\partial\left\langle\overline{u_{i}}\right\rangle^{f}}{\partial x_{j}}+\frac{\partial\left\langle\overline{u_{j}}\right\rangle^{f}}{\partial x_{i}}\right)\right] \\
-\phi\left(v_{f} K_{f i j}^{-1}+\phi_{f} b_{f i j}\left(\left\langle\overline{u_{k}}\right\rangle^{f}\left\langle\overline{u_{k}}\right\rangle^{f}\right)^{\frac{1}{2}}\right)\left\langle\overline{u_{i}}\right\rangle^{f}
\end{gathered}
$$

$$
\begin{aligned}
& \phi_{f} \rho_{f} c_{p f} \frac{\partial\langle\bar{T}\rangle^{f}}{\partial t}+\phi_{f} \rho_{f} c_{p f} \frac{\partial}{\partial x_{j}}\left\langle\overline{u_{j}}\right\rangle^{f}\langle\bar{T}\rangle^{f} \\
& =\frac{\partial}{\partial x_{i}}\left[\left(k_{f}+\frac{\rho_{f} c_{p f} v_{t f}}{\sigma}\right) \phi_{f} \delta_{i j}+\left(k_{t o r f}\right)_{i j}+\left(k_{d i s f}\right)_{i j}\right] \frac{\partial\langle\bar{T}\rangle^{f}}{\partial x_{j}} \\
& \quad+a_{f} h_{f}\left(\langle\bar{T}\rangle^{s}-\langle\bar{T}\rangle^{f}\right)
\end{aligned}
$$

$$
\begin{aligned}
& \frac{\partial\langle k\rangle^{f}}{\partial t}+\frac{\partial}{\partial x_{j}}\left\langle\overline{u_{j}}\right\rangle^{f}\langle k\rangle^{f} \\
& =\frac{\partial}{\partial x_{j}}\left[\left(v+\frac{v_{t}}{\sigma_{k}}\right)_{f} \delta_{i j}+\frac{\left(k_{d i s f}\right)_{i j}}{L e_{k f} \phi_{f} \rho_{f} c_{p f}}\right] \frac{\partial\langle k\rangle^{f}}{\partial x_{j}}
\end{aligned}
$$$$
+2 v_{t f}\left\langle s_{i j}\right\rangle^{f}\left\langle s_{i j}\right\rangle^{f}-\langle\varepsilon\rangle^{f}+\phi_{f}^{2} b_{f n}\left(\left\langle\overline{u_{j}}\right\rangle^{f}\left\langle\overline{u_{j}}\right\rangle^{f}\right)^{\frac{3}{2}}
$$ 


$$
\begin{gathered}
\frac{\partial\langle\varepsilon\rangle^{f}}{\partial t}+\frac{\partial}{\partial x_{j}}\left\langle\bar{u}_{j}\right\rangle^{f}\langle\varepsilon\rangle^{f} \\
=\frac{\partial}{\partial x_{i}}\left[\left(v+\frac{v_{t}}{\sigma_{\varepsilon}}\right)_{f} \delta_{i j}+\frac{\left(k_{d i s f}\right)_{i j}}{L e_{\varepsilon f} \phi_{f} \rho_{f} c_{p f}}\right] \frac{\partial\langle\varepsilon\rangle^{f}}{\partial x_{j}} \\
+\left(2 c_{1} v_{t f}\left\langle s_{i j}\right\rangle^{f}\left\langle s_{i j}\right\rangle^{f}-c_{2}\langle\varepsilon\rangle^{f}\right) \frac{\langle\varepsilon\rangle^{f}}{\langle k\rangle^{f}} \\
+c_{2} \phi_{f}^{2} b_{f n} \sqrt{\frac{c_{D} \phi_{f}}{2 K_{f n}}}\left(\left\langle\overline{u_{j}}\right\rangle^{f}\left\langle\overline{u_{j}}\right\rangle^{f}\right)^{2} \\
\left(1-\phi_{1}-\phi_{2}\right) \rho_{s} c_{s} \frac{\partial\langle\bar{T}\rangle^{s}}{\partial t}=\frac{\partial}{\partial x_{i}}\left(\left(1-\phi_{1}-\phi_{2}\right) k_{s} \delta_{i j}+\left(k_{t o r}\right)_{i j}\right) \frac{\partial\langle\bar{T}\rangle^{s}}{\partial x_{j}} \\
-a_{1} h_{1}\left(\langle T\rangle^{s}-\langle T\rangle^{1}\right)-a_{2} h_{2}\left(\langle T\rangle^{s}-\langle T\rangle^{2}\right)
\end{gathered}
$$

where

$$
\begin{gathered}
v_{t f}=c_{D} \frac{\left(\langle k\rangle^{f}\right)^{2}}{\langle\varepsilon\rangle^{f}},\left\langle s_{i j}\right\rangle^{f}=\frac{1}{2}\left(\frac{\partial\left\langle\bar{u}_{i}\right\rangle^{f}}{\partial x_{j}}+\frac{\partial\left\langle\bar{u}_{j}\right\rangle^{f}}{\partial x_{i}}\right) \text { and } \\
\langle a\rangle^{f}=\frac{1}{V_{f}} \int_{V_{f}} a d V
\end{gathered}
$$

The sub- and super-scripts $f(=1$ and 2$)$ and $s$ refer to hot fluid $(f=1)$, cold fluid $(f=2)$ and solid $(s)$, respectively. (Note that $\langle k\rangle^{f}$ is the intrinsic average of turbulence kinetic energy, which should not be confused with the thermal conductivity of fluid, $k_{f}$.) Moreover, $\phi_{f}=V_{f} / V$ is the fluid volume fraction while $L e_{k f}$ and $L e_{\varepsilon f}$ are the Lewis numbers for the mechanical dispersions. Moreover, $K_{f i j}, b_{f i j}$ and $h_{f}$ are the permeability tensor, Forchheimer tensor and 6 
interfacial heat transfer coefficient, respectively, and $K_{f n}^{-1}=K_{f i j}^{-1}\left\langle\bar{u}_{i}\right\rangle^{f}\left\langle\bar{u}_{j}\right\rangle^{f} /\left\langle\bar{u}_{k}\right\rangle^{f}\left\langle\bar{u}_{k}\right\rangle^{f}$ and $b_{f n}=b_{f i j}\left\langle\bar{u}_{i}\right\rangle^{f}\left\langle\bar{u}_{j}\right\rangle^{f} /\left\langle\bar{u}_{k}\right\rangle^{f}\left\langle\bar{u}_{k}\right\rangle^{f}$ are the directional permeability and Forchheimer constant. The resulting macroscopic equations, namely, (1), (2), (3), (4) and (5) for hot and cold fluids (i.e. $f=1$ and 2), and the heat conduction equation (6) for the solid phase, constitute a set of the three-energy equation model for analyzing complex turbulent flow and heat transfer encountered in heat exchangers. (For the case of laminar flow, only equations (1), (2), (3) and (6) may be considered with $v_{t f}=0$ everywhere.) These macroscopic equations may be utilized to analyze flow and temperature fields within any other complex assemblies consisting of small-scale elements in a clutter, which one does not want to grid. Note that the same set of the equations can be used also for clear fluid flows with one of the porosity $\phi_{1}=1$ and the other $\phi_{1}=0$, and for the conduction within a solid with $\phi_{1}=\phi_{2}=0$. In other words, the porous media concept allows us to cover not only convection in porous media but all other heat transfer modes from pure conduction to pure convection, simply by prescribing the spatial distribution of porosity (and that of the corresponding permeability).

\section{Subscale Model for Permeabilty Tensor}

In order to close and apply the foregoing set of the macroscopic equations based on VAT to a particular system, we must establish the subscale models for the permeability tensor $K_{f i j}$, Forchheimer tensor $b_{f i j}$, tortuosity tensor $\left(k_{t o r}\right)_{i j}$, dispersion tensor $\left(k_{d i s}\right)_{i j}$ and interfacial heat transfer coefficient $h_{f}$ for both hot and cold fluids (i.e. $f=1$ and 2). In what follows, we shall consider possible subscale models for describing these unknowns, considering the case of orthotropic structure in which the macroscopic flow makes angles $\alpha, \beta$ and $\gamma$ with the principle axes of the structure, as shown in Figure 2. In this paper, we focus on the subscale models for the permeability tensor, Forchheimer tensor and interfacial heat transfer, whereas possible subscale models for tortuosity tensor 
and dispersion tensor can be found elsewhere [Nakayama et al. [8]].



Figure 2. Typical structural element

Let us consider a macroscopically uniform laminar flow through a bank of infinitely long cylinders in yaw. As shown in Figure 3 for the $x-y$ plane, the cross section of the cylinder may be rectangular (as in a fin) or circle (as in a tube). The cylinder rows of the bank may be either aligned (as in the figure) or staggered. Such bank arrangements are relevant to various industrial applications such as cooling from extended fins and steam generation in tubular (and shell-and-tube) heat exchangers. In most bank configurations, flow and heat transfer condition stabilizes for a cylinder beyond the first few rows such that the flow and heat transfer characteristics under periodically fully-developed conditions are of particular interest. Thus, we shall consider an infinitely large bank to investigate the periodically fully-developed velocity and temperature fields. Such periodically fully-developed computations were performed for a bundle of isothermal square rods as shown in Figure 3. 


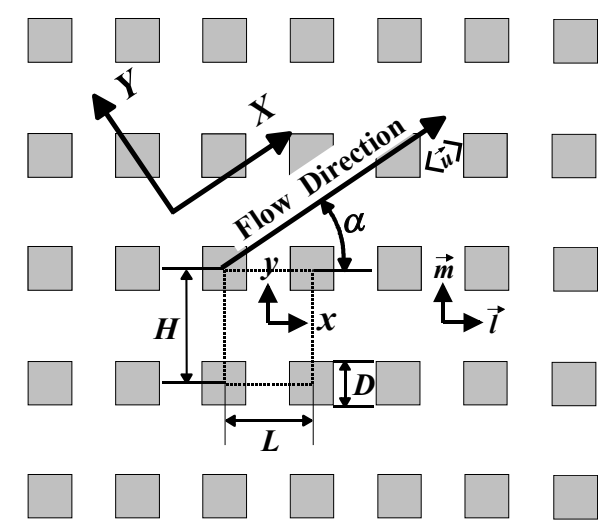

Figure 3 . A bundle of square rods $(\mathrm{z}=$ const plane $)$

The microscopic governing equations, namely, the equations of continuity, Navier-Stokes and energy equation subject to periodically fully-developed boundary conditions were numerically solved using well-known SIMPLE algorithm proposed by Patankar and Spalding [9]. Due to the periodicity, only one structural unit may be taken for a computational domain. Typical velocity and temperature fields obtained by both fully threedimensional scheme [Patankar [10]] and quasi-three-dimensional scheme [Nakayama et al. [11]] are shown for the case of $H / L=1$, $\alpha^{\prime}=45^{\circ}$ (projected angle), $\gamma=45^{\circ}$ (yaw angle), $R e_{L}=|\langle\vec{u}\rangle| L / v_{f}=600$ and $\operatorname{Pr}=1$ in Figure 4, where excellent agreement between two schemes is confirmed. Thus, the effective quasi-three-dimensional calculation procedure [Nakayama et al. [11]] has been used to cover a wide range of the parameters. The Reynolds number is varied from $10^{-2}$ to $6 \times 10^{3}$. Both cross flow angle $\alpha^{\prime}$ and yaw angle $\gamma$ are varied from 0 to $\pi / 2$ with an increment $\pi / 36$ to cover all possible macroscopic flow directions in the three-dimensional space, such that entire solution surfaces may be constructed over the domain $0 \leq \alpha^{\prime} \leq \pi / 2$ and $0 \leq \gamma \leq \pi / 2$. Moreover, the ratio $H / L$ is set to 1 , $3 / 2$ and 2 to investigate the effects of the degree of the anisotropy, whereas the ratio $D / L$ is fixed to $1 / 2$ for all calculations. In the following, these microscopic results are processed to establish the subscale models for flow resistance and heat transfer within the small-scale elements. 
As illustrated in Figure 2, a small structure may be regarded as an anisotropic porous medium, in which three principal axes $(\vec{l}, \vec{m}, \vec{n})$ are mutually orthogonal. Referring to these axes, the macroscopic velocity vector in an arbitrary direction may be presented by

$$
\langle\vec{u}\rangle=|\langle\vec{u}\rangle|(\cos \alpha \vec{l}+\cos \beta \vec{m}+\cos \gamma \vec{n})
$$

where the Darcian (apparent) velocity $\langle\vec{u}\rangle\left(=\phi\langle\vec{u}\rangle^{f}\right)$ is used in place of the intrinsic velocity $\langle\vec{u}\rangle^{f}$. Under the macroscopically uniform velocity as given by equation (8), the volume averaged momentum equation (2) reduces to

$$
-\frac{\partial\langle p\rangle^{f}}{\partial x_{i}}=\left(\mu_{f} K_{f}^{-1} i j+\rho_{f} b_{f i j}|\langle\vec{u}\rangle|\right)\left\langle u_{j}\right\rangle
$$

where $\left\langle u_{k}\right\rangle\left\langle u_{k}\right\rangle=|\langle\vec{u}\rangle|^{2}$. Thus, the macroscopic momentum equation leads to the Forchheimer extended Darcy's law [Forchheimer [12]], generalized for the case of anisotropic porous media.

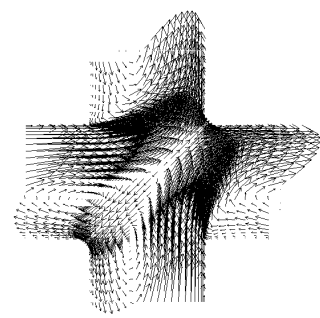

Velocity vectors on $z=$ const. plane

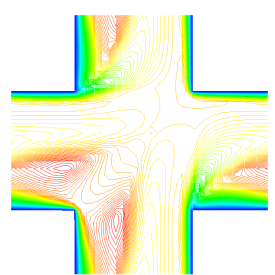

$w$ contours

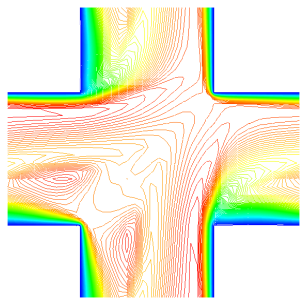

Isotherms

(a) Results based on the full three dimensional calculation procedure

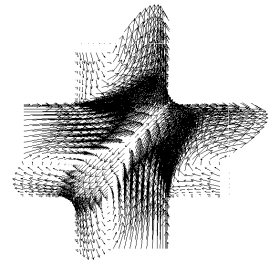

Velocity vectors on $z=$ const. plane $w$ contours

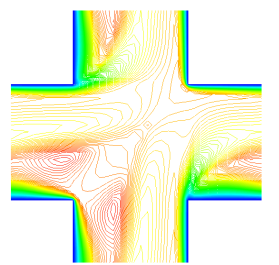

(b) Results based on the quasi-three dimensional calculation procedure

Figure 4. Comparison of two distinct three dimensional calculation procedures

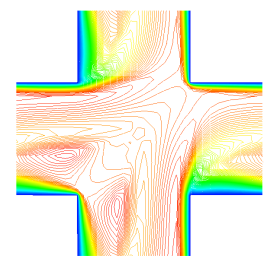

Isotherms 
Applications of the Volume Averaging Theory $\quad$ Mapana J Sci, 11, 3(2012)

$$
\left(H / L=1, \alpha^{\prime}=45^{\circ}, \gamma=45^{\circ}, \operatorname{Re}_{L}=600, \operatorname{Pr}=1\right)
$$

When the velocity (i.e. Reynolds number) is low, the proposed model equation (9) reduces to Darcy's law as

$$
-\frac{\partial\langle p\rangle^{f}}{\partial x_{i}}=\left(\mu_{f} K_{f}^{-1} i j+\rho_{f} b_{f i j}|\langle\vec{u}\rangle|\right)\left\langle u_{j}\right\rangle \cong \mu_{f} K_{f i j}^{-1}\left\langle u_{j}\right\rangle
$$

For the orthotropic media, the permeability tensor may be modeled following Dullien [1979] as

$$
K_{f i j}^{-1}=\left(l_{i} l_{j}\right) / K_{f 1}+\left(m_{i} m_{j}\right) / K_{f 2}+\left(n_{i} n_{j}\right) / K_{f 3}
$$

such that

$$
-\frac{\partial\langle p\rangle^{f}}{\partial x_{i}} \cong \mu_{f} K_{f}^{-1}{ }_{i j}\left\langle u_{j}\right\rangle=\left(\frac{\cos \alpha}{K_{f 1}} l_{i}+\frac{\cos \beta}{K_{f 2}} m_{i}+\frac{\cos \gamma}{K_{f 3}} n_{i}\right)|\langle\vec{u}\rangle|
$$

where

$$
\cos \alpha=\frac{l_{j}\left\langle u_{j}\right\rangle}{|\langle\vec{u}\rangle|}, \quad \cos \beta=\frac{m_{j}\left\langle u_{j}\right\rangle}{|\langle\vec{u}\rangle|}, \quad \cos \gamma=\frac{n_{j}\left\langle u_{j}\right\rangle}{|\langle\vec{u}\rangle|}
$$

Thus, the directional permeability measured along the macroscopic flow direction $s$ is given by

$$
\frac{1}{K_{f n}}=\frac{\cos ^{2} \alpha}{K_{f 1}}+\frac{\cos ^{2} \beta}{K_{f 2}}+\frac{\cos ^{2} \gamma}{K_{f 3}}
$$

such that

$$
-\frac{\partial\langle p\rangle^{f}}{\partial s}=\frac{\mu_{f}}{K_{f n}}|\langle\vec{u}\rangle|
$$

or, in dimensionless form, as

$$
-\frac{\partial\langle p\rangle^{f}}{\partial s} \frac{L^{2}}{\mu_{f}|\langle\vec{u}\rangle|}=\frac{L^{2}}{K_{f n}}
$$

The gradient of the intrinsic average pressure may readily be 
evaluated using the microscopic results as

$$
\begin{aligned}
& -\frac{\partial\langle p\rangle^{f}}{\partial s}=\frac{\cos \alpha}{L(H-D)} \int_{-(H-D) / 2}^{(H-D) / 2}\left(\left.p\right|_{x=-L / 2}-\left.p\right|_{x=L / 2}\right) d y \\
& +\frac{\cos \beta}{H(L-D)} \int_{-(L-D) / 2}^{(L-D) / 2}\left(\left.p\right|_{y=-H / 2}-\left.p\right|_{y=H / 2}\right) d y+\frac{\mu \cos \gamma}{\left(H L-D^{2}\right)} \prod_{P_{f}} \frac{\partial w}{\partial n} d P
\end{aligned}
$$

Thus, the directional permeability $K_{f_{n}}$ may readily be determined by reading the intercept of the ordinate variable, as we plot $\left.-\left(\partial\langle p\rangle^{f} / \partial s\right)\left(L^{2} / \mu_{f} \mid\langle\vec{u}\rangle\right)\right)$ against $R e_{L}$, as done in the previous study on the cross flow case [Nakayama et al. [13]].

The solution surfaces of the directional permeability are constructed using the numerical values and presented in terms of $L^{2} / K_{f_{n}}$ against the projected angle $\alpha^{\prime}$ and the yaw angle $\gamma$ for the cases of $H / L=1$ and $3 / 2$ in Figure $5(a)$. The solution surface changes drastically as the ratio $H / L$ departs from unity. It is interesting to note that the effect of the projected angle $\alpha^{\prime}$ on the directional permeability is totally absent for the arrangement $H / L=$ 1. The coefficients $K_{f_{1}} K_{f_{2}}$ and $K_{f_{3}}$ in the proposed expression (11) may be determined by fitting the numerical results against the solution surfaces based on equation (14). Such solution surfaces generated by the proposed equation (14) are presented in Figure 4 (b) for comparison. The numerical values of $K_{f_{1}} K_{f_{2}}$ and $K_{f_{3}}$ determined in this manner are listed in Table 1 . The validity of the proposed equation (11) with the values listed in Table 1 can be examined further by plotting $L^{2} / K_{f_{n}}$ as shown in Figure 6 for the case of $\gamma=\pi / 2$, where the fluid flows perpendicularly to the rods. It is seen that the numerical results closely follow the curves generated from equation (14). 
Applications of the Volume Averaging Theory Mapana J Sci, 11, 3(2012)

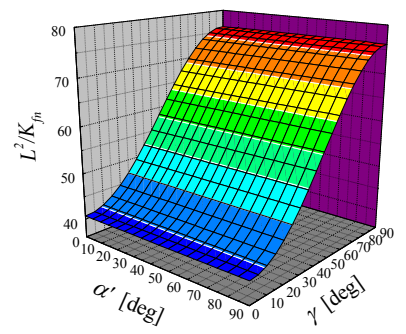

$H / L=1$



$H / L=3 / 2$

(a) Numerical experiments
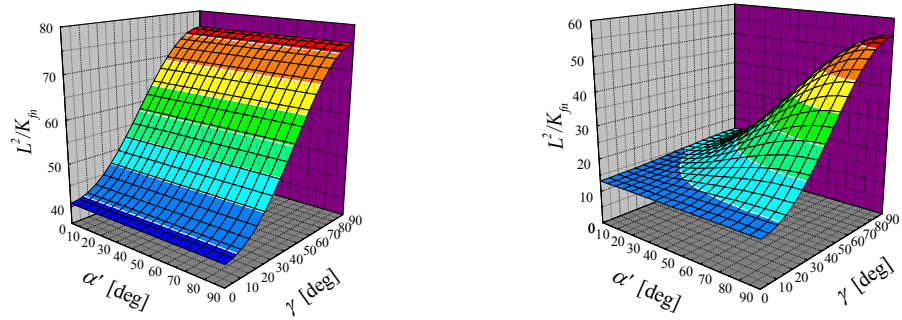

$H / L=1$

$H / L=3 / 2$

(b) Correlations

Figure 5. Solution surfaces for directional permeability 




Figure 6 Directional permeability at $\gamma=\pi / 2$

Table 1. Coefficients for macroscopic pressure gradient

\begin{tabular}{|c|c|c|c|c|c|c|}
\hline$H / L(\phi)$ & $L^{2} / K_{f_{1}}$ & $L^{2} / K_{f_{2}}$ & $L^{2} / K_{f_{3}}$ & $b_{f_{1}} L$ & $b_{f_{2}} L$ & $b b_{f_{1}} L$ \\
\hline \hline $1(0.750)$ & 76 & 76 & 41 & 0.2 & 0.2 & 8.2 \\
\hline \hline $3 / 2(0.833)$ & 16 & 55 & 13 & 0.1 & 0.6 & 3.2 \\
\hline \hline $2(0.875)$ & 7 & 42 & 6 & 0.05 & 0.8 & 1.2 \\
\hline
\end{tabular}

\section{Subscale Model for Forchheimer tensor}

When the velocity (i.e. Reynolds number) is sufficiently high, the Forchheimer term describing the form drag predominates over the Darcy term such that 
Applications of the Volume Averaging Theory Mapana J Sci, 11, 3(2012)

$$
-\frac{\partial\langle p\rangle^{f}}{\partial x_{i}}=\left(\mu_{f} K_{f}^{-1}{ }_{i j}+\rho_{f} b_{f i j}|\langle\vec{u}\rangle|\right)\left\langle u_{j}\right\rangle \cong \rho_{f} b_{f i j}|\langle\vec{u}\rangle|\left\langle u_{j}\right\rangle
$$

Usually, the principal axes of the permeability tensor $K_{f i j}^{-1}$ do not coincide with those of the Forchheimer tensor $b_{f_{i j}}$. For the orthotropic media in consideration, however, the tensors $b_{f_{i j}}$ should be symmetric and they must also satisfy the following symmetric conditions:

$$
\left.\frac{\partial b_{f n}}{\partial \alpha}\right|_{\alpha=0, \frac{\pi}{2}}=\left.\frac{\partial b_{f n}}{\partial \beta}\right|_{\beta=0, \frac{\pi}{2}}=\left.\frac{\partial b_{f n}}{\partial \gamma}\right|_{\gamma=0, \frac{\pi}{2}}=0
$$

where

$$
b_{f n} \equiv b_{f i j} \frac{\left\langle u_{i}\right\rangle\left\langle u_{j}\right\rangle}{|\langle\vec{u}\rangle|^{2}}
$$

is the directional Forchheimer coefficient measured along the macroscopic flow direction $s$. One of the simplest functions that satisfy these conditions may be:

$$
\begin{aligned}
b_{f i j}= & b_{f 1}\left(l_{i} l_{j}\right)+b_{f 2}\left(m_{i} m_{j}\right)+b_{f 3}\left(n_{i} n_{j}\right) \\
& +b b_{f 1} \cos \alpha \cos \beta\left(\left(l_{i} m_{j}\right)+\left(l_{j} m_{i}\right)\right) \\
& +b b_{f 2} \cos \beta \cos \gamma\left(\left(m_{i} n_{j}\right)+\left(m_{j} n_{i}\right)\right) \\
& +b b_{f 3} \cos \gamma \cos \alpha\left(\left(n_{i} l_{j}\right)+\left(n_{j} l_{i}\right)\right)
\end{aligned}
$$

which results in

$$
\begin{aligned}
b_{f n}= & b_{f 1} \cos ^{2} \alpha+b_{f 2} \cos ^{2} \beta+b_{f 3} \cos ^{2} \gamma \\
& +2 b b_{f 1} \cos ^{2} \alpha \cos ^{2} \beta+2 b b_{f 2} \cos ^{2} \beta \cos ^{2} \gamma \\
& +2 b b_{f 3} \cos ^{2} \gamma \cos ^{2} \alpha
\end{aligned}
$$


such that

$$
-\frac{\partial\langle p\rangle^{f}}{\partial s}=\frac{\mu_{f}}{K_{f n}}|\langle\vec{u}\rangle|+\rho_{f} b_{f n}|\langle\vec{u}\rangle|^{2}
$$

or, in dimensionless form, as

$$
-\frac{\partial\langle p\rangle^{f}}{\partial s} \frac{L}{\rho_{f} \mid\langle\vec{u}\rangle^{2}}=\frac{L^{2}}{K_{f n} R_{L}}+b_{f n} L
$$

Plotting the results of macroscopic pressure gradient in terms of $-\left(\partial\langle p\rangle^{f} / \partial s\right)\left(L / \rho_{f}|\langle\vec{u}\rangle|^{2}\right)$ and reading the horizontal asymptotes, we can readily determine the directional Forchheimer constant.

The numerical values of the directional Forchheimer constant for the cases of $H / L=1$ and $3 / 2$ are shown in terms the solution surfaces of $b_{f_{n}} L$ in Figures 7(a). These figures clearly show that, for fixed $\gamma$, the directional Forchheimer constant attains its peak around $\alpha^{\prime}=\pi / 2$, while, for fixed $\alpha^{\prime}$, it decreases monotonically from $\gamma=\pi / 2$ to 0 .



$H / L=1$

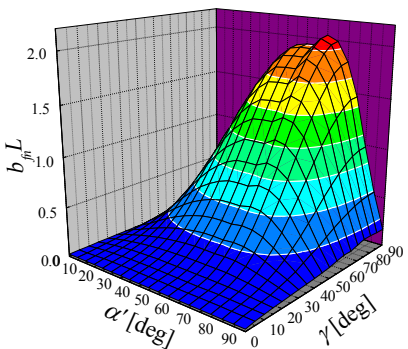

$H / L=3 / 2$

(a) Numerical experiments 


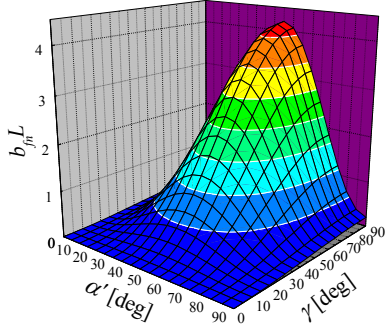

$H / L=1$



$H / L=3 / 2$

(b) Correlations

Figure 7. Solution surfaces for directional Forchheimer coefficient

From this observation, we find that the coefficients and $b b_{f 1}$ is nonzero while $b_{f_{3}}, b b_{f 2}$ and $b b_{f 3}$ in equation (22) should vanish for the bank of cylinders, such that

$$
\begin{aligned}
b_{f n} & =b_{f 1} \cos ^{2} \alpha+b_{f 2} \cos ^{2} \beta+2 b b_{f 1} \cos ^{2} \alpha \cos ^{2} \beta \\
& =\left(b_{f 1} \cos ^{2} \alpha^{\prime}+b_{f 2} \sin ^{2} \alpha^{\prime}+2 b b_{f 1} \cos ^{2} \alpha^{\prime} \sin ^{2} \alpha^{\prime} \sin ^{2} \gamma\right) \sin ^{2} \gamma
\end{aligned}
$$

The corresponding $b_{f_{n}} L$ surfaces based on the proposed expression (25) with the values of $b_{f_{1}} b_{f_{2}}$ and $b b_{f_{1}}$ as listed in Table 1 are presented in Figure 7(b) for comparison. Furthermore, the numerical results of the directional Forchheimer constant obtained with $\gamma=\pi / 2$ for $H / L=1,3 / 2$ and 2 are presented in Figure 8 as a function of the cross flow angle $\alpha\left(=\alpha^{\prime}\right)$. In the same figure, the solid curves generated from the proposed equation (25) are presented to elucidate the validity of the proposed expression. Note that, for this case of $\gamma=\pi / 2$, the foregoing equation reduces to

$b_{f n}=b_{f 1} \cos ^{2} \alpha+b_{f 2} \sin ^{2} \alpha+2 b b_{f 1} \cos ^{2} \alpha \sin ^{2} \alpha$

It is interesting to note that the numerical results for the cases 
$H / L=3 / 2$ and 2 show two consecutive peaks, while the model equation (26) yields only one peak (the first peak). The second peak appears when the macroscopic flow angle $\alpha$ reaches roughly $\tan ^{-1}(H / L)$. Note that, for the case of $H / L=1$, this second peak coincides with the first one. Unfortunately, the model equation is incapable of describing the second peak.

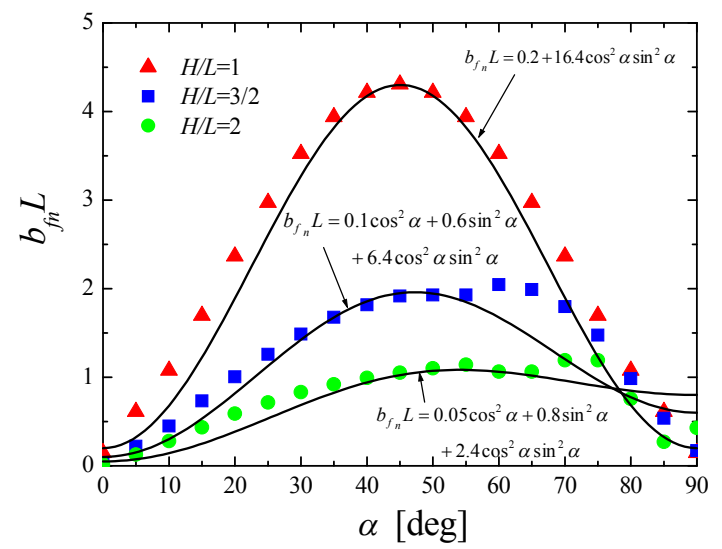

Figure 8. Directional Forchheimer coefficient at $\gamma=\pi / 2$



Figure 9. Dimensionless macroscopic pressure gradient 
Zukauskas [14] assembled the experimental data for the fullydeveloped pressure drop across the tube banks in both inlinesquare and staggered-triangle arrangements, and presented a chart for the Euler number (i.e. the dimensionless macroscopic pressure gradient). His inline-square arrangement corresponds to the present arrangement with $\alpha=0, \gamma=\pi / 2$ and $L / D=2$. However, it is noted that, in reality, the macroscopic flow direction rarely coincides with the principal axes, since even small disturbances at a sufficiently high Reynolds number make the flow deviate from the axis. Thus, it is understood that the chart provided by Zukauskas gives only the average level of the pressure drop within a range of small $\alpha$ (say $0^{\circ}<\alpha<5^{\circ}$ ). The dimensionless macroscopic pressure gradient $-\left(\partial\langle p\rangle^{f} / \partial s\right)\left(L / \rho_{f}|\langle\vec{u}\rangle|^{2}\right)$ for the case of $\gamma=\pi / 2$ and $L / D=2$ is plotted against $R e_{L}$ in Figure 9, where the curves generated from the model equation (24) with the numerical values taken from Table 1 and Figure 8 (note that $b_{f n} L=0.2$ and 0.6 , for $\alpha=0^{\circ}$ and $5^{\circ}$, respectively) are drawn together with the empirical chart provided by Zukauskas for the inline-square arrangement. The agreement between these curves appears fairly good.

\section{Subscale Model for Interfacial Heat Transfer}

According to Kuwahara et al. [15], the interfacial heat transfer coefficient may be obtained by substituting the microscopic temperature results into the following equation:

$$
h_{f} \equiv \frac{\frac{1}{V} \int_{A_{\text {int }}} k_{f} \nabla T \cdot d \vec{A}}{\left(\langle T\rangle^{S}-\langle T\rangle^{f}\right)}=\frac{\frac{1}{A_{f l u i d}} \int_{P_{\text {int }}}\left(-k_{f} \frac{\partial T}{\partial n}\right) d P}{\left(\langle T\rangle^{S}-\langle T\rangle^{f}\right)}
$$

where $A_{\text {int }}$ is the total interface between the fluid and solid, while $d \vec{A}$ is its vector element pointing outward from the fluid side to solid side. In Figure 10, the heat transfer results obtained at $\alpha=0$ and $\pi / 4$ for the cross flows (i.e. $\gamma=\pi / 2$ ) are presented in terms of the interfacial Nusselt number $N u_{L}=h_{f} L / k_{f}$ against the Reynolds number $R e_{L}$. 




Figure 10. Effect of Reynolds number on directional Nusselt number $(\operatorname{Pr}=1)$

The figure suggests that the lower and higher Reynolds number data follow two distinct limiting lines for the case of non-zero $\alpha$, namely, $\alpha=\pi / 4$. The lower Reynolds number data stay constant for the given array and flow angle, whereas the high Reynolds number data vary in proportion to $R e_{L}{ }^{0.6}$. Another series of computations changing the Prandtl number, conducted following Kuwahara et al. [15], revealed that the exponents associated with the Reynolds and Prandtl numbers are the same as those Wakao and Kaguei [16] observed as collecting and scrutinizing reliable experimental data on interfacial convective heat transfer coefficients in packed beds. The similarity, albeit the difference in the Reynolds number dependence, between the Nusselt number $N u_{L}$ and the macroscopic pressure gradient as given by equation (23) is noteworthy, which prompts us to model the directional Nusselt number as follows:

$$
N u_{L} \equiv \frac{h_{f} L}{k}=c_{f}+d_{f} \operatorname{Re}_{L}^{0.6} \operatorname{Pr}_{f}^{1 / 3}
$$


The experimental correlation proposed by Zukauskas [17] for the heat transfer from the circular tubes in staggered banks are compared with the present results obtained for the case of $\alpha=\pi / 4, \gamma=\pi / 2$ and $H / L=1$. (Note $N u_{f} \cong N u_{L} / 2$ and $R e_{f} \cong R e_{L}$ in equation (39) of Zukauskas since $D / L=1 / 2$.) The present results follow closely along the experimental correlation of Zukauskas as increasing the Reynolds number. Grimson [18, 19] carried out an exhaustive experiment to investigate heat transfer from tube rows of a bank in both staggered and aligned arrangements with respect to the direction of the macroscopic flow. His case, in which the ratio of the transverse pitch to tube diameter and that of the longitudinal pitch to tube diameter are 3 and 1.5, respectively, gives a configuration close to the present orthogonal configuration with $\alpha=\pi / 4, \gamma=\pi / 2$ and $H / L=1$. Thus, the experimental correlation established by Grimson for the case is also presented in the figure, which agrees very well with the present numerical results. These correlations are believed to hold for a comparatively wide Reynolds number range, covering from a predominantly laminar flow regime to turbulent flow regime.

Following the procedure similar to the one adopted for determining the directional permeability, the coefficient $\left.c_{f} \equiv N u_{L}\right|_{R e_{L} \rightarrow 0}$ for each macroscopic flow angle is evaluated and plotted in terms of the solution surfaces in Figures 11 (a), using the low Reynolds number data. It is noted that the effect of the projected angle $\alpha^{\prime}$ on the interfacial heat transfer coefficient is totally absent for the arrangement $H / L=1$. The similarity between the solution surfaces of $c_{f}$ and those of $L^{2} / K_{f n}$ is obvious, which leads us to introduce a functional form as follows:

$$
c_{f}=\left(c_{f 1}{ }^{n_{c}} \cos ^{2} \alpha+c_{f 2}{ }^{n_{c}} \cos ^{2} \beta+c_{f 3}{ }^{n_{c}} \cos ^{2} \gamma\right)^{1 / n_{c}}
$$

such that $c_{f}$ reduces to $c_{f_{1}}, c_{f_{2}}$ and $c_{f_{3}}$ for $\alpha=0, \beta=0$ and $\gamma=0$, respectively, as it should. Careful examination of the numerical results over the whole domain within $0 \leq \alpha^{\prime} \leq \pi / 2$ and 
$0 \leq \gamma \leq \pi / 2$ suggests that $n_{c}$ is close to minus one, which leads us to a harmonic mean expression as

$$
\frac{1}{c_{f}}=\frac{\cos ^{2} \alpha}{c_{f 1}}+\frac{\cos ^{2} \beta}{c_{f 2}}+\frac{\cos ^{2} \gamma}{c_{f 3}}
$$

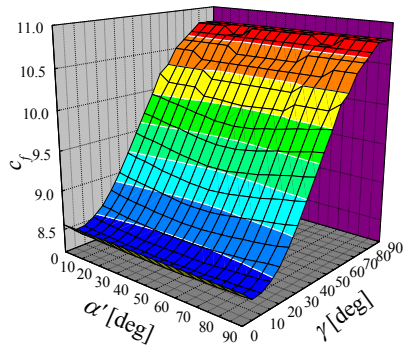

$H / L=1$

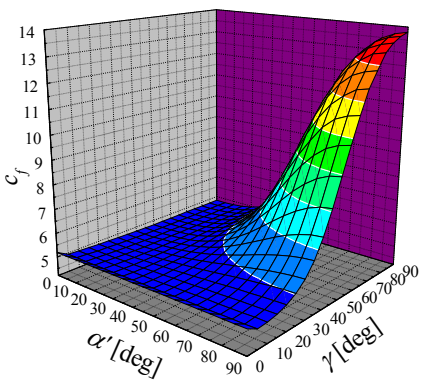

$H / L=3 / 2$

(a) Numerical experiments

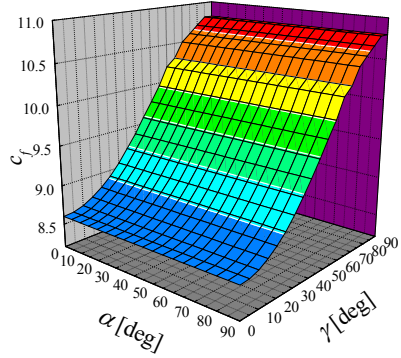

$H / L=1$

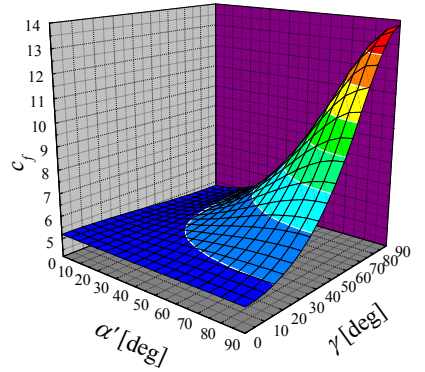

$H / L=3 / 2$

(b) Correlations

Figure 11. Solution surfaces for directional heat transfer coefficient at small Reynolds number. 
Applications of the Volume Averaging Theory Mapana J Sci, 11, 3(2012)

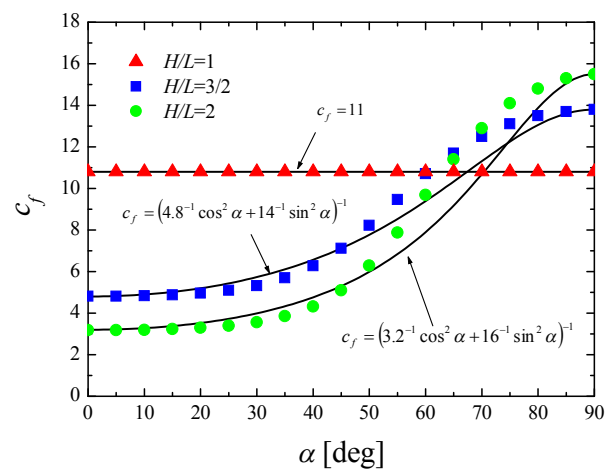

Figure 12. Effect of the cross flow angle $\alpha$ on the coefficient $c_{f}$ at $\gamma=\pi / 2$

The values of $c_{f_{1}}, c_{f_{2}}$ and $c_{f_{3}}$ listed in Table 2 have been determined by fitting the numerical results against the foregoing equation. The resulting surfaces based on the proposed expression (30) are presented in Figure 11(b) for their comparison with the surfaces based on the numerical experiments shown in Figure 11(a). Furthermore, Figure 12 shows the numerical results of $c_{f}$ obtained at $\gamma=\pi / 2$ for the three distinct arrangements, namely, $H / L=1,3 / 2$ and 2 . The solid curves in the figure are generated from the proposed equation (30) with the values of $c_{f_{1}}$ and $c_{f_{2}}$ as listed in Table 2.

Table 2. Coefficients for Directional Nusselt number

\begin{tabular}{|c|c|c|c|c|c|c|}
\hline$H / L(\phi)$ & $c_{f_{1}}$ & $c_{f_{2}}$ & $c_{f_{3}}$ & $n_{c}$ & $d_{f_{1}}=d_{f_{2}}$ & $n_{d}$ \\
\hline \hline $1(0.750)$ & 11 & 11 & 8.6 & -1.0 & 0.90 & 4.5 \\
\hline \hline $3 / 2(0.833)$ & 4.8 & 14 & 5.2 & -1.0 & 0.77 & 4.5 \\
\hline \hline $2(0.875)$ & 3.2 & 16 & 3.6 & -1.0 & 0.67 & 4.5 \\
\hline
\end{tabular}

The second coefficient $d_{f}$ may be determined using the data $N u_{L} / \operatorname{Re}_{L}{ }^{0.6} \operatorname{Pr}_{f}{ }^{1 / 3}$ in the high Reynolds number range. The 
resulting solution surfaces of $d_{f}$ are presented in Figure 13 for $H / L=1$ and $3 / 2$. Unlike the Forchheimer coefficient $b_{f_{n}}$, the coefficient $d_{f}$ stays roughly constant for a fixed yaw angle $\gamma$.

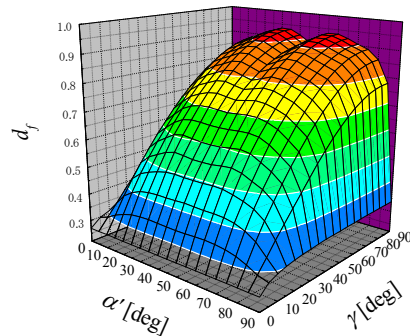

$H / L=1$

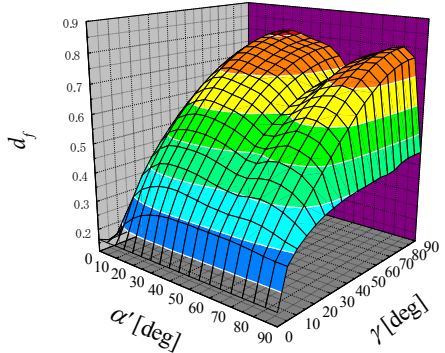

$H / L=3 / 2$

(a) Numerical experiments

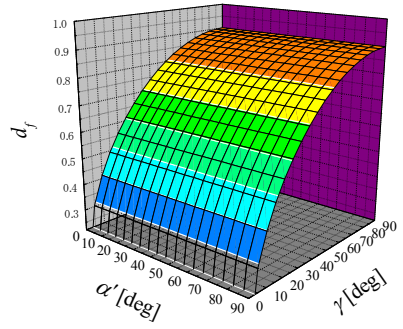

$H / L=1$

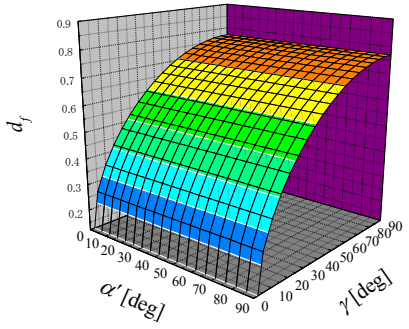

$H / L=3 / 2$

(b) Correlations

Figure 13. Solution surfaces for directional heat transfer coefficient at large Reynolds number.

Somewhat more careful observation on the solution surfaces reveals that the coefficient $d_{f}$ drops abruptly as the projected angle $\alpha^{\prime}$ reaches close to either 0 or $\pi / 2$ (in which the fluid flows along the principal axis of the structure). However, as already pointed out, it is quite unlikely to have the macroscopic flow align perfectly with the principal axes. Thus, we may assume that $d_{f}$ is the function of the yaw angle $\gamma$ alone, namely, $d_{f}=d_{f}(\gamma)$. It is 
interesting to note that $d_{f}=d_{f}(\gamma)$ is consistent with the idea of the effective velocity $u_{\text {eff }}=|\langle\vec{u}\rangle| \sin \gamma$ used in the hot-wire anemometry. Thus, we may model $d_{f}$ as

$$
d_{f}=\left(d_{f 1}{ }^{n_{d}} \sin ^{2} \gamma+d_{f 3}{ }^{n_{d}} \cos ^{2} \gamma\right)^{1 / n_{d}}
$$

A careful observation on the solution surfaces leads us to $d_{f_{3}} \cong 0$, and also reveals the values of $d_{f_{1}}$ and $n_{d}$ as listed in Table 2. Thus, we propose the expression as follows:

$$
\begin{aligned}
N u_{L}= & \left(c_{f 1}{ }^{n^{c}} \cos ^{2} \alpha+c_{f 2}{ }^{n_{c}} \cos ^{2} \beta+c_{f 3^{n^{c}}} \cos ^{2} \gamma\right)^{1 / n_{c}} \\
& +d_{f 1} \sin ^{\frac{2}{n_{d}}} \gamma \operatorname{Re}_{L}{ }^{0.6} \operatorname{Pr}_{f}^{1 / 3}
\end{aligned}
$$

or

$$
\begin{aligned}
N u_{D}= & \frac{1}{2}\left(c_{f 1}{ }^{n_{c}} \cos ^{2} \alpha+c_{f 2}{ }^{n_{c}} \cos ^{2} \beta+c_{f 3}{ }^{n_{c}} \cos ^{2} \gamma\right)^{1 / n_{c}} \\
& +\frac{d_{f 1}}{2^{0.4}} \sin ^{\frac{2}{n_{d}}} \gamma \operatorname{Re}_{D}{ }^{0.6} \operatorname{Pr}_{f}^{1 / 3}
\end{aligned}
$$

Note that the exponents $n_{c}=-1$ and $n_{d}=9 / 2$ irrespectively of the value of $H / L$, while the coefficients $c_{f_{1}}, c_{f_{2}} c_{f_{3}}$ and $d_{f_{1}}$ depend on that particular geometrical configuration.

Zukauskas [14] investigated the effect of the yaw angle on the interfacial heat transfer rate. He varied the yaw angle $\gamma$ for both staggered and aligned arrangements, and compared the corresponding heat transfer rates for the same Reynolds number. He pointed out that the data when normalized by the value obtained at $\gamma=\pi / 2$ for all staggered and inline arrangements, namely, $N u_{D} /\left.N u_{D}\right|_{\gamma=\pi / 2}$, can be approximated by a single curve irrespectively of the Reynolds number. His data for both staggered 
and inline arrangements are plotted in Figure 14 together with the expression based on the model equation (32b), namely,

$$
\frac{N u_{D}}{\left.N u_{D}\right|_{\gamma=\pi / 2}} \cong \sin ^{2 / n_{d}} \gamma=\sin ^{4 / 9} \gamma
$$

for the case of sufficiently high Reynolds number. The agreement between the experimental data and the curve based on equation (33) is fairly good, which indicates the validity of the model equation (32b). It should also be noted that the staggered arrangement corresponds to the case of $\alpha^{\prime}=\pi / 4$ while the inline arrangement to the case in which $\alpha^{\prime}$ is close to zero (but $\alpha^{\prime} \neq 0$ since the macroscopic flow direction never coincides with the principal axis of the structure). Thus, these experimental data substantiates our finding based on the numerical experiment, namely, that the multiplicative constant for the interfacial Nusselt number $d_{f}$ stays virtually constant (irrespectively of $\alpha^{\prime}$ ) for a fixed yaw angle, as illustrated by the solution surfaces in Figure 13 .

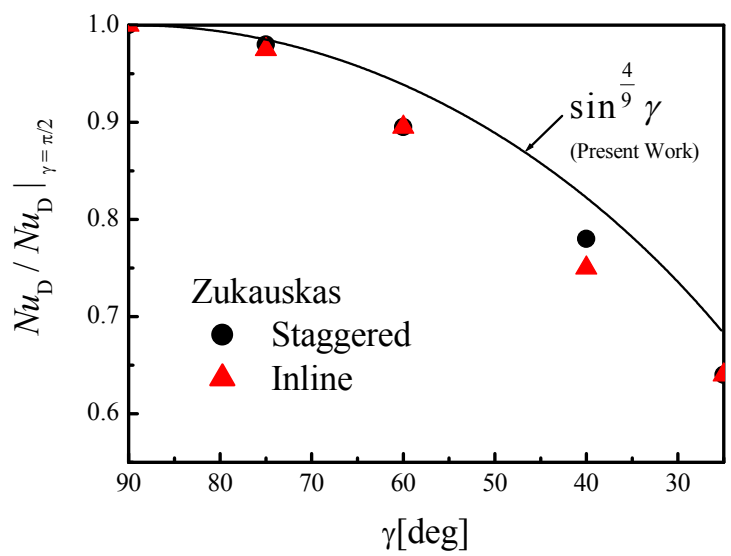

Figure 14. Effect of the yaw angle $\gamma$ on the interfacial Nusselt number

\section{Preliminary Application to Complex Heat Transfer Equipment}

To illustrate possible engineering applications, the foregoing macroscopic equations and their subscale models are exploited to 26 
analyze various heat and fluid flow systems such as a plate fin heat exchanger and a cross flow tubular heat exchanger as shown in Figure 15.

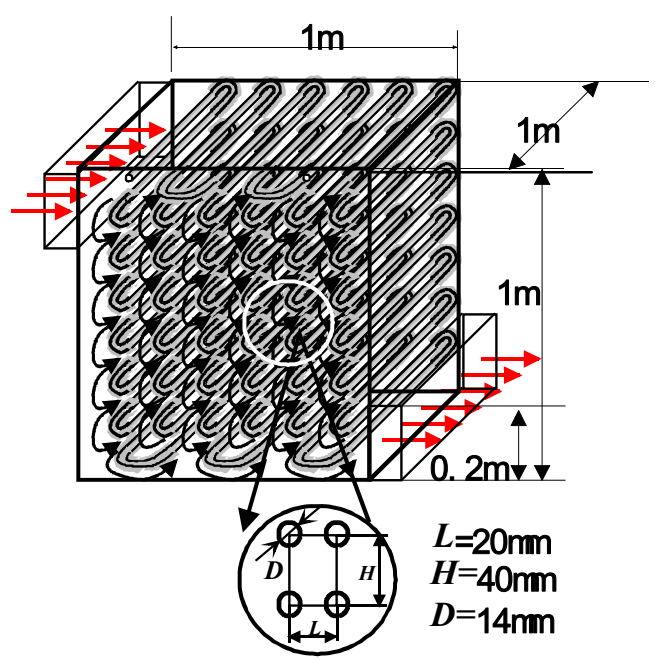

Figure 15. Cross flow tubular heat exchanger

The hot gas at $230 C^{\circ}$ enters from the upper left inlet at the uniform velocity $\left(\left(\dot{m} c_{p}\right)_{1}=4700 \mathrm{~W} / \mathrm{K}\right)$ and goes out from the lower right exit, while the water $\left(\left(\dot{m} c_{p}\right)_{2}=4700 \mathrm{~W} / \mathrm{K}\right)$ at $30 C^{\circ}$ passes through a coiled tube of inner diameter $14 \mathrm{~mm}$ to be heated up. It is assumed that the gas flow is laminar whereas the water flow in the tube is turbulent. Only the macroscopic equations (1), (2) and (3) for the gas phase $(f=1)$ are solved numerically along with the subscale models established beforehand for the permeability, Forcheimer tensors and interfacial heat transfer coefficient for the rod arrangement shown in Figure 15. The water flow, on the other hand, is assumed to follow Dittus-Boelter formula, and its bulk temperature along the tube is obtained solving the one-dimensional energy balance equation using the surrounding gas temperature available in that instant. Both gas and water temperatures are coupled to get converged temperature fields. The macroscopic temperature fields of the gas and water on the center plane are displayed in Figures 16(a) and (b), respectively. The figures clearly illustrate heat transfer from the hot gas to cold water. Naturally, heat transfer in 
the region close to the gas entrance is most significant.

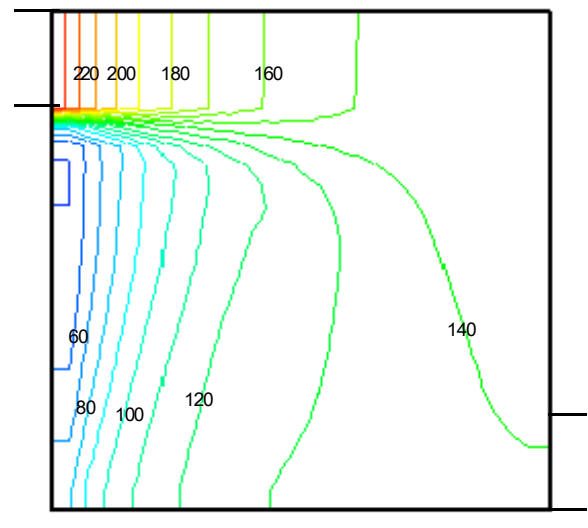

(a) Gas temperature

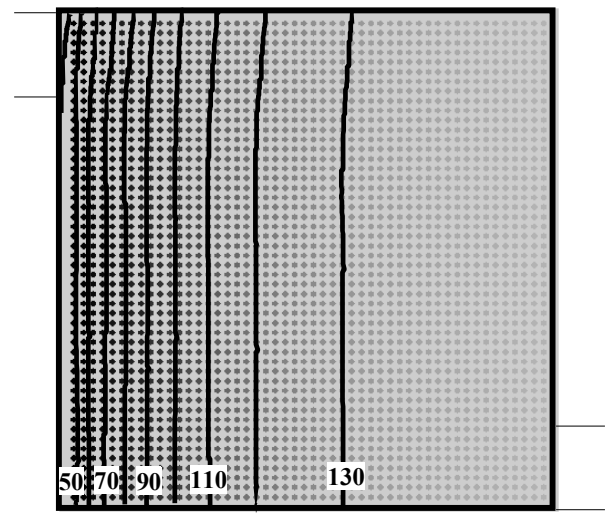

(b) Water temperature

Figure 16. Cross flow tubular heat exchanger

\section{Conclusions}

A systematic modelling procedure based on the theory of porous media is proposed to attack heat and fluid flow in complex manmade structures consisting small elements. A general set of macroscopic governing equations is derived exploiting the volume averaging theory. The sub-scale modeling and microscopic numerical treatments for its closure are proposed on the basis of the anisotropic porous media theory. As for illustrative applications, extensive numerical experiments are conducted for three-dimensional heat and fluid flow through a bank of cylinders in yaw, which represents a numerical model for manmade structures such as plate fin heat exchangers, and also for gas and water flows in a cross flow tubular heat exchanger. The porous media concept discussed here is not limited to complex heat transfer equipment, but has a great potential for further advances in complex heat transfer systems and for further exploration of emerging new technologies. Its applications to composting systems, bioheat transfer and cryoablation treatments have been carried out. 
Applications of the Volume Averaging Theory $\quad$ Mapana J Sci, 11, 3(2012)

\section{References}

[1] P Cheng, Heat transfer in geothermal systems. Advances in Heat Transfer, vol. 14, pp. 1-105, 1978.

[2] M Quintard and S Whitaker, One and two equation models for transient diffusion in two-phase systems, Advances in Heat Transfer, vol. 23, pp. 269-464, 1993.

[3] A Nakayama, PC-Aided Numerical heat transfer and convective flow, Boca Raton: CRC Press, 1995.

[4] P E DesJardin, Private communication, 2001.

[5] A Nakayama, F Kuwahara, A Naoki and G Xu, A three-energy equation model based on a volume averaging theory for analyzing complex heat and fluid flow in heat exchangers, Proc. Int. Conf. Energy Conversion and Application, Wuhan, China, ICECA'2001, pp.506-512, 2001.

[6] A Nakayama and F Kuwahara, Numerical modeling of convective heat transfer in porous media using microscopic structures, K Vafai, ed. Handbook of Porous Media, Marcel Dekker, Inc., New York, pp. 441-488, 2000.

[7] A Nakayama and F Kuwahara, A macroscopic turbulence model for flow in a porous medium, J. Fluids Engineering, vol. 121, pp. 427-433, 1999.

[8] A Nakayama, F Kuwahara and Y Kodama, An equation for thermal dispersion flux transport and its mathematical modeling for heat and fluid flow in a porous medium, J. Fluid Mechanics, vol. 563, pp. 81-96, 2006.

[9] S V Patankar and D B Spalding, A calculation procedure for heat, mass and momentum transfer in three-dimensional parabolic flows, Int. J. Heat Mass Transfer, vol. 15, pp.1787-1806, 1972.

[10] S V Patankar, Numerical Heat Transfer and Fluid Flow, Washington, DC: Hemisphere, 1980.

[11] A Nakayama, F Kuwahara and T Hayashi, Numerical modeling for three-dimensional heat and fluid flow through a bank of cylinders in yaw, J. Fluid Mechanics, vol. 498, pp. 139-159, 2004.

[12] P H Forchheimer, Wasserbewegung durch Boden, Z. Ver. Dtsch. Ing., vol. 45, pp. 1782-1788, 1901.

[13] A Nakayama, F Kuwahara, T Umemoto and T Hayashi, Heat and fluid flow within an anisotropic porous medium, J. Heat Transfer, vol. 124, pp. 746-753, 2002. 
[14] A Zukauskas and Konvektivnyi Perenos v Teploobmennikakh, Convective transfer in heat exchangers, Nauka, Moscow, p. 472, 1982.

[15] F Kuwahara, M Shirota and A Nakayama, A numerical study of interfacial convective heat transfer coefficient in two-energy equation model for convection in porous media, Int. J. Heat Mass Transfer, vol. 44, pp. 1153-1159, 2001.

[16] N Wakao and S Kaguei, Heat and mass transfer in packed beds, Gorden and Breach Sci. Publishers, New York, pp. 243-295, 1982.

[17] A Zukauskas, Heat transfer from tubes in crossflow, Advances in Heat Transfer, vol. 18, pp. 87-159, 1987.

[18] E D Grimson, Correlation and utilization of new data on flow resistance and heat transfer for cross flow of gases over tube banks, Transaction of ASME, vol. 59, pp. 583-594, 1937.

[19] E D Grimson, Heat transfer and flow resistance of gases over tube banks, Transaction of ASME, vol. 58, pp. 381-392, 1938. 